\title{
Too Much of a Good Thing? An Evolutionary Theory to Explain the Role of Ceramides in NAFLD
}

\author{
Annelise M. Poss and Scott A. Summers* \\ Department of Nutrition and Integrative Physiology, University of Utah, Salt Lake City, UT, United States
}

Non-alcoholic fatty liver disease (NAFLD), which ranges from the relatively benign and reversible fatty liver (NAFL) to the more advanced and deadly steatohepatitis (NASH), affects a remarkably high percentage of adults in the population. Depending upon severity, NAFLD can increase one's risk for diabetes, cardiovascular disease, and hepatocellular carcinoma. Though the dominant histological feature of all forms of the disease is the accumulation of liver triglycerides, these molecules are likely not pathogenic, but rather serve to protect the liver from the damaging consequences of overnutrition. We propose herein that the less abundant ceramides, through evolutionarily-conserved actions intended to help organisms adapt to nutrient excess,

OPEN ACCESS

Edited by:

Ondrej Šeda,

Charles University, Czechia

Reviewed by:

Christa Buechler,

University Medical Center

Regensburg, Germany Giovanni Solinas,

University of Gothenburg, Sweden

*Correspondence:

Scott A. Summers scott.a.summers@health.utah.edu

Specialty section:

This article was submitted to Diabetes: Molecular Mechanisms, a section of the journal Frontiers in Endocrinology

Received: 11 May 2020 Accepted: 24 June 2020

Published: 31 July 2020

Citation:

Poss AM and Summers SA (2020) Too Much of a Good Thing? An Evolutionary Theory to Explain the Role of Ceramides in NAFLD.

Front. Endocrinol. 11:505. doi: 10.3389/fendo.2020.00505 drive the cellular events that define NAFL/NASH. In early stages of the disease process, they promote lipid uptake and storage, whilst inhibiting utilization of glucose. In later stages, they stimulate hepatocyte apoptosis and fibrosis. In rodents, blocking ceramide synthesis ameliorates all stages of NAFLD. In humans, serum and liver ceramides correlate with the severity of NAFLD and its comorbidities diabetes and heart disease. These studies identify key roles for ceramides in these hepatic manifestations of the metabolic syndrome.

Keywords: fatty liver, ceramide, NASH, NAFL, NAFLD (non alcoholic fatty liver disease)

\section{INTRODUCTION}

Non-alcoholic fatty liver disease (NAFLD) is reaching epidemic proportions, affecting $25 \%$ of the adult population worldwide $(1,2)$. It consists of two disease states of varying severity: the relatively benign simple fatty liver termed non-alcoholic fatty liver (NAFL) and the more severe non-alcoholic steatohepatitis (NASH). NASH can lead to hepatic scarring (cirrhosis) and fibrosis, which contribute to hepatocellular carcinoma (HCC), the most common liver cancer and the third leading cause of cancer-related death in the USA. Moreover, NAFLD increases one's risk for cardiometabolic disorders including type-2 diabetes and coronary artery disease. These interrelated cardiometabolic disorders account for a sizable proportion of global deaths and overall health expenditures.

The defining feature of NAFL is the accumulation of large lipid droplets in hepatocytes. $\mathrm{NASH}$ is a worsened condition characterized by additional hepatocellular ballooning, lobular inflammation, and fibrosis. These histological characteristics of NASH reveal serious tissue damage that can result in organ failure. Though the most striking histological feature of NAFLD across all stages is the accumulation of triglycerides, these lipids are likely inert markers of the condition, rather than being bioactive drivers of the pathology. Instead, studies have identified 
roles for sphingolipids such as ceramides in each stage of NAFLD and in many features of NASH: fat accumulation, insulin resistance, mitochondrial dysfunction, apoptosis, and fibrosis. The purpose of this review is to discuss the relevant evidence in both human and animal studies for the role of ceramides in each element of these liver pathologies. Moreover, we propose a unifying, evolutionary theory to explain why ceramides initiate these deleterious actions.

\section{SPHINGOLIPID BIOSYNTHESIS AND DEGRADATION}

Sphingolipids are a richly diverse lipid class, comprising over 4,000 distinct species that serve a wide variety of biological roles. Their de novo synthesis starts in the endoplasmic reticulum with the condensation of fatty and amino acids to produce the basic sphingoid scaffold. A series of enzymatic reactions follow that produce ceramides, which are the precursors for the more abundant sphingolipids (e.g., sphingomyelins, gangliosides). Sphingolipids play integral roles in membrane structure and fluidity as well as cellular growth and function, including initiation of a coordinated stress response and ultimately apoptosis (3). Circulating factors associated with metabolic disorders including saturated fats, inflammatory cytokines, and glucocorticoids invariably stimulate biosynthesis of ceramides $(4,5)$.

The first enzyme in the biosynthetic pathway is serine palmitoyl transferase (SPT), which condenses amino (e.g., serine) and fatty acids (e.g., palmitoyl-CoA) to produce the sphingoid backbone (6). The third step in the pathway, catalyzed by one of six different (dihydro)ceramide synthases (CERS1-6), accounts for much of the diversity in sphingolipids by adding acyl chains to the sphingoid scaffold (7). These enzymes show variable substrate specificities and tissue distributions. CERS2 is the primary isoform in the liver, adding the C24 and C24:1 acylchains. These very-long-chain ceramides appear benign. CERS5 and 6 add the C16-acyl chains, producing the ceramides that are most strongly implicated in cardiometabolic diseases. CERS6 produces C16-ceramides that contribute to NAFLD and adipose tissue dysfunction $(8,9)$. CERS5 appears to have a deleterious role in the heart (10). Other articles in this review series will offer a more in-depth analysis of the unique roles and distributions of distinct ceramide species.

The product of the CERS reactions, during de novo biosynthesis, are the dihydroceramides. Dihydroceramide desaturases introduce a double bond into the $\mathrm{d} 4$ position of the sphingoid backbone of dihydroceramides to produce the more abundant ceramides (11). Despite the structural similarities between ceramides and dihydroceramides, differing by only a double bond, the two molecules exhibit radically different functional roles in cellular signaling and metabolism $(12,13)$. Mammals contain two desaturases: a ubiquitously expressed DES1 and a skin- and gut-specific DES2 that inserts an additional hydroxyl group in the $\mathrm{d} 4$ position to generate phytoceramides.

Following their synthesis in the ER, ceramides and dihydroceramides traffic to the Golgi apparatus where they are converted into complex sphingolipids through the addition of various head groups (e.g., phosphocholine to produce sphingomyelin; sugar moieties to produce the glucosylceramides and gangliosides; phosphate to produce ceramide-1-phosphate; or acyl-CoAs to produce the 1-O-acylceramides). Ceramides can be re-formed by the hydrolysis of the choline head group from sphingomyelins or through a salvage pathway that involves the re-acylation of sphingosine (via CERS enzymes) produced by ceramidase-mediated ceramide degradation $(14,15)$. Receptors for adiponectin, an anti-diabetic and cardioprotective adipokine, are ligand-activated ceramidases $(16,17)$. Studies in rodents have demonstrated hepatoprotective effects of adiponectin in multiple modes of liver injury. Moreover, adiponectin is an independent risk factor for NAFLD in diverse clinical cohorts (18-22). The rate of adiponectin-stimulated ceramide degradation thus appears to influence the progression of NAFLD and other cardiometabolic diseases.

Experimental manipulations of the enzymes that control ceramide synthesis or metabolism have produced a wealth of data about the role of ceramides in in the progressive stages of NAFL and $\mathrm{NASH}$, as well as associated comorbidities including insulin resistance and cardiovascular disease. These will be discussed in depth below.

\section{CERAMIDES IN NAFL}

NAFL, or simple fatty liver, is characterized in humans by $\geq 5 \%$ macrovesicular fat accumulation in hepatocytes without substantial inflammation or hepatocellular damage (1). NAFL is considered relatively benign and is asymptomatic. Studies demonstrate no direct relationship between the extent of steatosis and survival in humans (23). In the current progressive model of NAFLD development, however, NAFL precedes the more severe liver disease states such as NASH, cirrhosis and HCC.

Though increased hepatic triglyceride deposition precedes $\mathrm{NASH}$, triglycerides are likely inert storage molecules that do not directly elicit the cellular dysfunction that causes NASH $(24,25)$. In accordance with this theory, inhibiting triglyceride synthesis at the level of DGAT2 reduces steatosis, but enhances fibrosis, oxidative stress, and lipid peroxidation $(26,27)$. The bioactive ceramides are more likely to drive the pathology. In accordance with this theory, a number of studies have identified correlations between ceramides and different measures of NAFLD/NASH in humans (28-37).

Interventional studies in rodents invariably demonstrate that ceramides are necessary for NAFL development. One such study was performed using mice that allow for conditional depletion of the Degs1 gene that encodes DES1. Deletion of the gene allows the investigator to acutely replace the ceramides in tissues with dihydroceramides lacking the key double bond (13). Deleting Degs1 from adult mice either prevented or reversed hepatic steatosis in $o b / o b$ mice by blunting lipid uptake (via the fatty acid translocase CD36) and decreasing expression of lipogenic genes (e.g., Srebf1). Similarly, liver-specific ablation of the Degs1 gene encoding DES1 (or liver-targeted knockdown of Degs1 using shRNA) reversed high fat diet-induced hepatic steatosis 


\section{A Ceramides in NAFL}

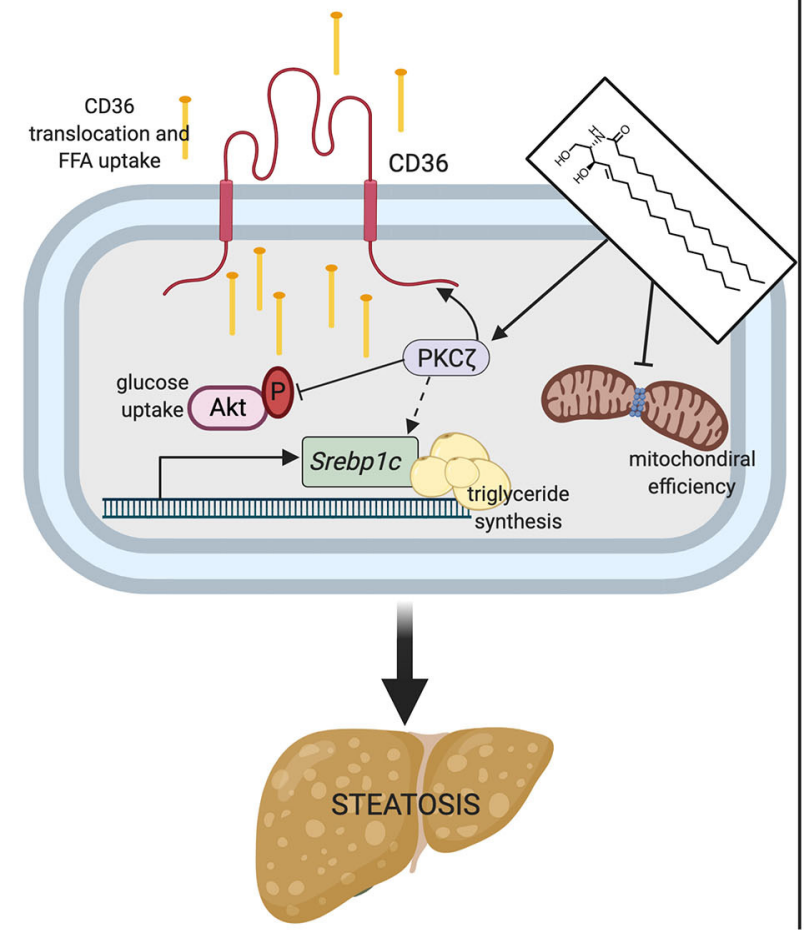

B Ceramides in NASH/Fibrosis

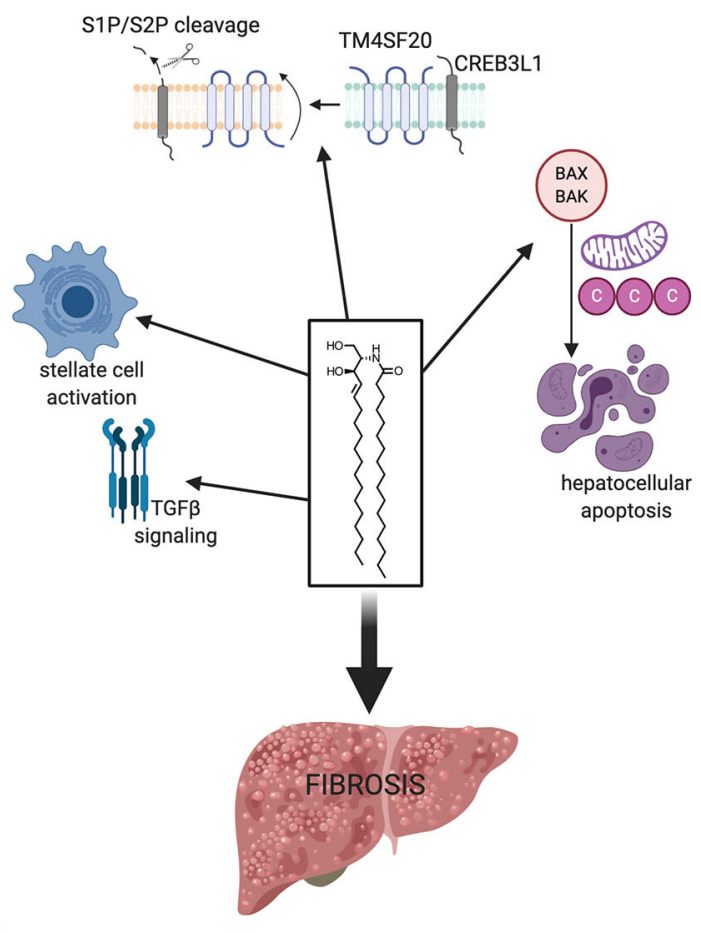

FIGURE 1 | Mechanisms of ceramides contribute to NALF and NASH/fibrosis. (A) In NAFL, ceramides promote lipid accumulation ultimately resulting in hepatosteatosis. In promoting steatosis, ceramides stimulate the translocation of CD36 to its active site on the plasma membrane via obligate intermediate PKC $\zeta$ where it facilitates uptake and esterification of free fatty acids. Ceramide action on $\mathrm{PKC} \zeta$ also upregulates transcription of Srebpc1 and inhibits Akt. Ceramides also reduce mitochondrial efficiency, promoting fat as a preferred substrate. (B) Ceramides promote fibrosis through TGF- $\beta$ signaling, CREB3L1 proteolysis, stellate cell activation, and hepatocellular apoptosis. Srebpc1, sterol regulatory element binding protein 1; S1P/S2P, site 1 protease, site 2 protease; TM4SF20, transmembrane 4 super family 20; CREB3L1, CAMP responsive element binding protein 3. Figure was created with BioRender.com.

(13). Similar findings were reported using mice that allow for inducible, liver-specific overexpression of acid ceramidase (38) or liver-specific deletion of CERS6 (9), which lower ceramides, normalize lipid uptake and metabolism, and resolve diet-induced hepatic steatosis. Thus, depleting ceramides from the liver, by either blocking their synthesis or stimulating their degradation, resolves NAFL. In each of these studies, the interventions also resolved other features of the metabolic syndrome including insulin resistance and serum hypertriglyceridemia.

Though the studies in the preceding paragraph have demonstrated that ceramides are necessary for NAFL, relatively few have evaluated whether they are sufficient to drive the pathology using gain-of-function approaches. In vivo, the closest has been studies by the Gonzalez laboratory, which found that intestinal Farnesoid X receptors induced ceramides which traveled to the liver to stimulate steatosis. In these studies, they administered C16-ceramides to antibiotic-treated mice, finding that they induced steatosis and increased expression of the lipogenic gene Srebp1c and other transcriptional markers of steatosis (e.g., Cidea, Fasn, etc.) (39). In vitro, overexpression of CERS6 overexpression in primary hepatocytes was shown to be sufficient to increase levels of C16-ceramides, induce triglyceride accumulation, impair mitochondrial respiration, and antagonize insulin signaling (40).

\section{CERAMIDES, LIPID UPTAKE, AND TRIGLYCERIDE SYNTHESIS}

Several mechanisms explain the ceramide-induction of hepatic steatosis (Figure 1A).

First, ceramides increase lipid uptake into the liver, at least in part by stimulating the translocation of the CD36 fatty acid translocase to the plasma membrane $(13,38)$. This event involves the atypical protein kinase C zeta (PKC $\zeta)$ (38), a ceramide effector that has several roles in lipid and glucose metabolism. Addition of exogenous short-chain ceramide analogs activates PKC $\zeta$ in H4IIe hepatocytes and overexpression of dominant negative $\mathrm{PKC} \zeta$ negates ceramide-induced CD36 translocation. In some tissues, this ceramide-PKC $\zeta$ axis also impairs glucose utilization through diminished activation of Akt (protein kinase B) (41). 
A

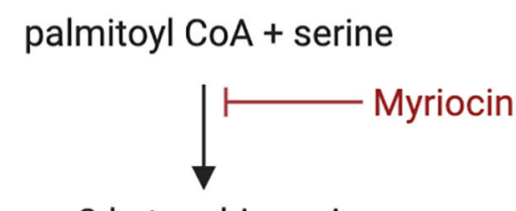

3-ketosphinganine

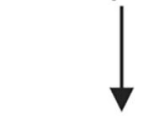

sphinganine

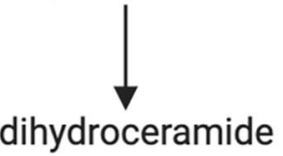

dihydroceramide

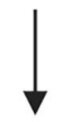

ceramide в Mechanisms of Myriocin in NAFLD

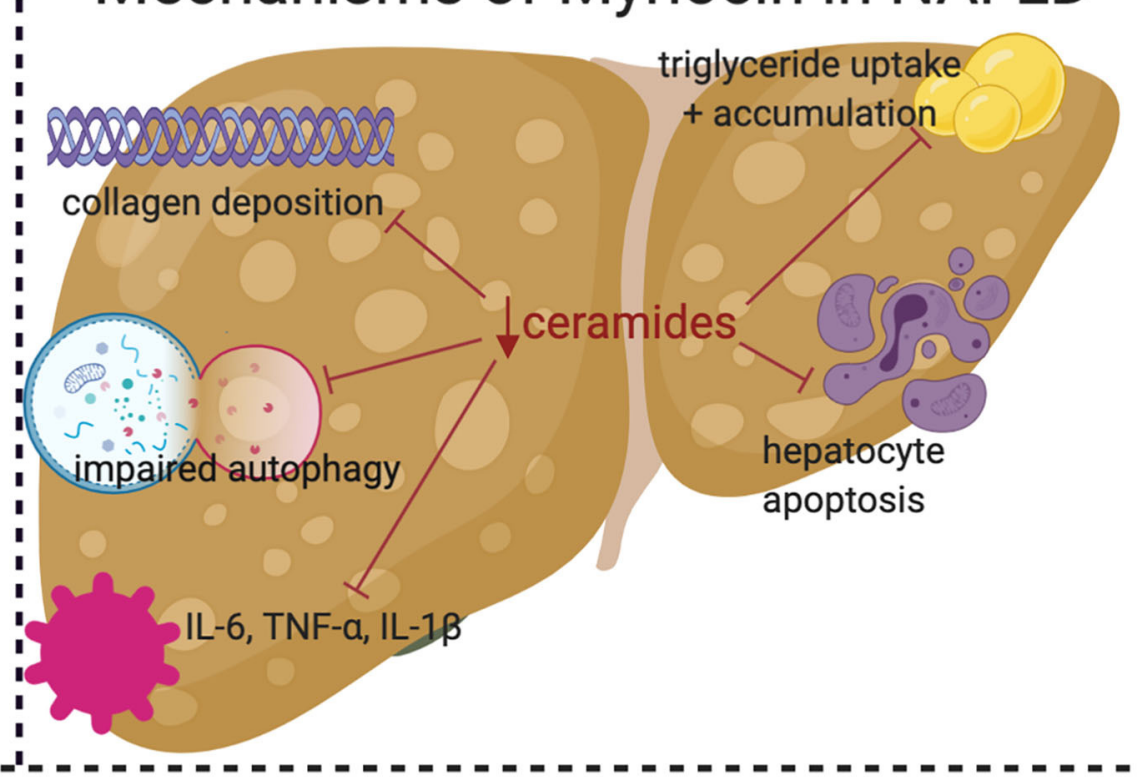

FIGURE 2 | Pharmacological inhibition of de novo ceramide synthesis prevents high fat diet (HFD) induced NASH. (A) Simplified schematic of ceramide de novo synthesis pathway, demonstrating the target of Myriocin (in red). (B) Graphical summary of the beneficial effects of Myriocin treatment in HFD-fed Sprague-Dawley rats. HFD-induced NAFLD related effects are shown in black with the beneficial effects of Myriocin treatments shown with red lines. Figure was created with BioRender.com.

Second, ceramides activate signaling pathways that induce triglyceride synthesis, in part by inducing the master transcriptional regulator sterol response element binding protein (SREBP). These are potently induced by ceramides and during NAFLD pathogenesis (39). In fact, gene variants and polymorphisms of Srebpf1 are associated with increased risk of NAFLD development (42). In vitro, ceramides are sufficient to stimulate hepatic Srebpf1 gene expression, activating a number of downstream targets that facilitate triglyceride production and fatty acid elongation (43). This phenomenon is independent of intracellular cholesterol levels, mediated by ceramides regulating posttranscriptional physiological processing of Srebpf1. Similarly, liver specific DES1 depletion in mice displays a striking downregulation of SREBPs (13). This mechanism is likely driven through the aforementioned ceramide-PKC $\zeta$ axis, which is established as a contributor to hypertriglyceridemia and hepatic steatosis in mice (44). Indeed, several studies have shown that $\mathrm{PKC} \zeta$ regulates SREBP-mediated triglyceride synthesis.

\section{NAFL TO NASH TRANSITION/NASH}

The transition from NAFL to NASH worsens an individual's prognosis, as NASH is considerably more likely to progress to HCC and cirrhosis, and ultimately mortality, than the relatively benign NAFL $(1,45)$. Meta-analyses have also identified fibrosis grade as an independent risk factor for liver and cardiovascularrelated mortality (46). Understanding this transition is essential for developing clinical interventions to prevent end stage liver disease and improve patient outcomes. NASH differs from NAFL in that steatosis is accompanied by lobular inflammation, hepatocellular ballooning degeneration, and fibrosis. NAFLD has a complicated bidirectional relationship with insulin resistance, which is considered a driving factor for progression to more severe liver disease states (47).

NASH patients have elevated liver ceramides and increased hepatic expression of ceramide synthesizing genes $(29,30,32$, $48,49)$. Ceramides have also been observed as a signature of the NASH disease state in disparate mouse models of the condition, including those that are not obese. For example, an unbiased comparison of two diet-mediated mouse NASH models, the methionine choline deficient and the atherogenic diets, identified alterations in sphingolipid metabolism as a common feature in both (34). This study included a thorough exploration of histological, transcriptional, and lipidomic changes in both models. Despite considerable differences in the mechanisms of these two NASH models, both exhibited decreases in cholesterogenesis, transcriptional upregulation of ceramide synthases, and increased de novo sphingolipid synthesis resulting in elevated ceramides, dihydroceramides, and glucosylceramides. In accordance, NASH patients that undergo a weight loss-based lifestyle intervention that reverses the condition show a reduction in circulating ceramides and hepatic expression of pro-ceramide synthesizing genes (32).

In rodents, several studies have shown that pharmacological reduction of ceramides prevents NASH onset (Figure 2). The majority have utilized myriocin, which is a potent and irreversible inhibitor of serine palmitoyltransferase. One such 
study used high fat diet feeding in adult Sprague-Dawley rats and demonstrated that myriocin prevented steatosis and fibrosis (50). In this experiment, ceramide depletion led to inactivation of signaling by c-Jun $\mathrm{N}$-terminal kinase (JNK), a kinase implicated in inflammatory responses and the regulation of apoptosis. Moreover, myriocin normalized levels of inflammatory cytokines such as TNF- $\alpha$, IL-1 $\beta$, and IL- 6 . A similar study, also conducted in Sprague-Dawley rats fed a high fat diet, demonstrated that myriocin normalized body weight, serum transaminase, and serum triglycerides (28). Studies in cultured hepatocytes showed that myriocin normalized expression profiles of genes involved in fatty acid metabolism and restored autophagy. A final study performed in Wistar rats demonstrated that myriocin treatment for 7 days following establishment of high fat diet induce NAFLD was sufficient to lower ceramides, improve whole body insulin tolerance, and reduce hepatic steatosis (51). Ceramides are also elevated in livers of mice exposed to carbon tetrachloride, a toxin that is commonly used to mimic NASH, fibrosis, and cirrhosis (52).

Unfortunately, no studies involving direct manipulation of ceramide synthesizing genes to increase or decrease ceramides in the context of NASH have been published to date.

\section{CERAMIDES, CELL DEATH, AND FIBROSIS}

Apoptosis is an essential element in the cascade of events that cause NASH. In both in vitro and in vivo models, administration of pan-caspase inhibitors or targeted genetic ablation of specific caspases suppresses apoptosis, and fibrosis (53). Ceramides have long-been known to induce apoptosis, as they increase mitochondrial outer membrane permeability to cytochrome C, leading to caspase activation (54). Blocking ceramide production negates the pro-apoptotic effects of many different cellular insults (55), including the saturated fatty acids and cytokines implicated in NASH. Ceramides have also been demonstrated to induce hepatocellular necrosis through induction of mitochondrial failure (56).

Ceramides alter mitochondrial membrane permeability by several different mechanisms. They recruit the pro-apoptotic protein BAX to mitochondria, where it oligomerizes and increases membrane permeability (57). Ceramides bind voltage-dependent anion channel 2, which further increases mitochondrial outer membrane permeability (58). Ceramides, through extensive hydrogen bonding with themselves, are capable of forming channels in membranes that allow passage of small proteins (59-62). And, ceramides inhibit pro-survival signaling by the protein kinase Akt/PKB (63). This latter effect on Akt/PKB accounts for the ability of ceramides to induce insulin resistance $(63,64)$.

Ceramides may also directly participate in the fibrosis signaling pathways that control collagen deposition (Figure 1B). In fact, hepatic stellate cells, the major extracellular matrix producing cells of the liver, exhibit increased ceramide concentrations and ceramide synthesizing gene transcripts upon activation in culture (65). This suggests that bioactive lipids such as ceramides may affect hepatic stellate cell activation and hepatic collagen deposition during NASH. Though the precise mechanisms remain elusive, ceramides are positive regulators of signaling by TGF $\beta$, a major pro-fibrogenic cytokine (66-68). In addition, ceramides enhance cleavage of plasma membrane resident CREB3L1 by site- 1 or site- 2 proteases, a process that liberates a protein fragment that enters the nucleus to bind Smad4 and activate transcription of genes required for assembly of collagen-containing extracellular matrix $(69,70)$. This process, termed regulated intramembrane proteolysis, results from ceramides altering the orientation of TM4SF20, a protein that, in the absence of ceramides, blocks access of the proteases to CREB3L1 (70, 71).

\section{CERAMIDES IN NAFLD ASSOCIATED COMORBIDITIES}

NALFD is the hepatic manifestation of the metabolic syndrome. Ranging from a simple fatty liver to the far more severe NASH, the conditions increase risk for diabetes, cardiovascular disease, hepatocellular carcinoma and liver failure. In fact, cardiovascular disease is the most common cause of death in NAFLD patients, accounting for more than twice the number of deaths than progressive liver related complications $(1,72)$. Even in the absence of metabolic syndrome, NAFLD patients experience increased risk of cardiovascular disease (73).

Studies with knockout mice reveal that the liver provides $>50 \%$ of serum ceramides (13). Interestingly, measurement of ceramides and related sphingolipids in serum has proven to have clinical utility, serving as potent, cholesterol-independent prognostic biomarkers of cardiovascular disease incidence and mortality (74-79). Circulating ceramides have also been associated with insulin resistance and type 2 diabetes in large clinical cohorts (80-82). Though the literature is largely devoid of large studies relating circulating ceramides to NAFLD, the noted relationships between serum and hepatic ceramides as with established NAFLD and as a distinction between NAFL and NASH strongly suggests that their measurement could have utility for diagnosing the condition $(30,32,33)$.

A large proportion of patients with NAFL express a common gene variant (i.e., rs738409) in Patatin-like phospholipase domain-containing protein 3 (PNPLA3). The mutation in this triglyceride lipase gene leads to marked changes in liver fat. Unlike individuals with the "metabolic NAFLD" described in this review, those with PNPLA3-induced NAFLD often remain metabolically healthy, with less insulin resistance, diabetes, and cardiovascular disease $(83,84)$. Interestingly, ceramide concentrations are not elevated in individuals with "PNPLA3 NAFLD." The authors attributed the lack of hepatic ceramide in subjects with the PNPLA3 variant to their ability to effectively store fat as triglycerides, rather than letting it be diverted into the sphingolipid pathway. Notably, PNPLA3 mutant patients can still develop severe fibrosis (85), suggesting ceramideindependent modes of fibrogenesis exist, in addition to ceramidedependent ones.

Collectively, these studies indicate that ceramides are markers of NAFLD that play causative roles in several disease features. 
While more work is needed to determine the precise role of ceramides in NASH pathogenesis, the data thus far strongly support development of ceramide-lowering strategies to combat these liver pathologies.

\section{AN EVOLUTIONARY BASIS FOR THE ROLE OF CERAMIDES IN NAFLD}

We've discussed a number of ceramide-driven mechanisms that influence the health of the hepatocyte under conditions of nutrient overload. We speculate that ceramides serve as nutritional signals, accumulating when the triglyceride stores are saturated and the cellular energy needs are met. The cells respond to the increasing ceramides by initiating actions which protect them from detergent-like FFAs (86). Indeed, each of the ceramide mechanisms we have identified protect cellular membranes from the destabilizing actions of these lytic fatty acids.

In the early stages of disease progressions (e.g., NAFL), ceramides initiate actions that promote the safe uptake of fatty acids through the cellular membrane and facilitate their storage as triglycerides. For example, they increase the allotment of fatty acid translocases (e.g., CD36 or fatty acid binding proteins) on the cellular membrane, which facilitates safe passage of FFAs through the bilayer and speeds their esterification. In parallel, ceramides induce SREBP and its target genes (e.g., DGATs) to enable the incorporation of fatty acids into triglycerides. Ceramides also reduce glucose utilization by inhibiting Akt/PKB, thus allowing cells to switch to fatty acids as a preferred energy source. Lastly, they decrease mitochondrial efficiency, thus maximizing the number of fatty acids that can be oxidized while minimizing the effect on mitochondrial membrane potential. All of these actions, which were at one time likely intended to protect the cell from fatty acid toxicity, underlie NAFL.

As ceramide levels continue to rise, they initiate actions to protect the organism from the compromised, lipid-laden cells. For example, we hypothesize that ceramide-mediated apoptosis and fibrosis are extensions of the aforementioned signaling mechanisms that protect the organism when cells are compromised. For example, ceramide induction of apoptosis allows for a controlled cell death, preventing the release of cytosolic content into the extracellular space. Similarly, the

\section{REFERENCES}

1. Tesfay M, Goldkamp WJ, Neuschwander-Tetri BA. NASH: the emerging most common form of chronic liver disease. Mo Med. (2018) 115:225-9.

2. Ye Q, Zou B, Yeo YH, Li J, Huang DQ, Wu Y, et al. Global prevalence, incidence, and outcomes of non-obese or lean non-alcoholic fatty liver disease: a systematic review and meta-analysis. The lancet. Gastroenterology \& Hepatology (2020). doi: 10.1016/s2468-1253(20)30077-7

3. Nikolova-Karakashian MN, Rozenova KA. Ceramide in stress response. $A d v$ Exp Med Biol. (2010) 688:86-108. doi: 10.1007/978-1-4419-6741-1_6

4. Summers SA. Ceramides in insulin resistance and lipotoxicity. Prog Lipid Res. (2006) 45:42-72. doi: 10.1016/j.plipres.2005.11.002

5. Summers SA, Nelson DH. A role for sphingolipids in producing the common features of type 2 diabetes, metabolic syndrome X, Cushing's syndrome. Diabetes. (2005) 54:591-602. doi: 10.2337/diabetes.54.3.591

6. Merrill AH Jr. De novo sphingolipid biosynthesis: a necessary, but dangerous, pathway. J Biol Chem. (2002) 277:25843-6. doi: 10.1074/jbc.R200009200 induction of fibrosis allows the organism to protect itself from a damaged region of tissue. Thus, ceramides protect the organism from damage resulting from uncontrolled membrane lysis.

This theory-that ceramides signal cellular events to combat the burden of toxic levels of free fatty acids-provides a unifying framework and testable hypotheses about their role in disease.

\section{CONCLUSIONS}

The published data thus far clearly establish roles for ceramides as drivers of different features NAFLD. Nonetheless, additional work is needed. First, more comprehensive assessments of the roles of ceramides in the terminal steps of NASH (e.g., apoptosis and fibrosis) in rodent models are warranted. These should include investigations of whether ceramide-lowering is sufficient to reverse the pathology at the later stages of disease progression. Moreover, it should include genetic manipulations to discern which effects are due to autonomous actions within the hepatocyte. Second, additional clinical verifications, including lipidomics of large liver biobanks and genomic determinations of genes that influence ceramides, would support efforts to develop this therapeutic approach. Despite these areas of need, the evidence is strong that ceramides contribute to NAFLD. Therapeutic approaches that selectively lower ceramides could show enormous progress as a means of combating NAFLD. Ceramides merit a sustained and rigorous evaluation from the scientific community.

\section{AUTHOR CONTRIBUTIONS}

AP and SS drafted the manuscript in collaboration with one another. AP generated the figures. All authors contributed to the article and approved the submitted version.

\section{FUNDING}

The authors received research support from the National Institutes of Health (DK115824, DK116888, and DK116450 to SS), the Juvenile Diabetes Research Foundation (JDRF 3-SRA2019-768-A-B to SS), the American Diabetes Association (to SS), the American Heart Association (to SS), and the Margolis Foundation (to SS).

7. Cingolani F, Futerman AH, Casas J. Ceramide synthases in biomedical research. Chem Phys Lipids. (2016) 197:2532. doi: 10.1016/j.chemphyslip.2015.07.026

8. Raichur S, Brunner B, Bielohuby M, Hansen G, Pfenninger A, Wang B, et al. The role of C16:0 ceramide in the development of obesity and type 2 diabetes: CerS6 inhibition as a novel therapeutic approach. Mol Metab. (2019) 21:36-50. doi: 10.1016/j.molmet.2018.12.008

9. Turpin SM, Nicholls HT, Willmes DM, Mourier A, Brodesser S, Wunderlich $\mathrm{CM}$, et al. Obesity-induced CerS6-dependent C16:0 ceramide production promotes weight gain and glucose intolerance. Cell Metab. (2014) 20:67886. doi: 10.1016/j.cmet.2014.08.002

10. Russo SB, Baicu CF, Van Laer A, Geng T, Kasiganesan H, Zile MR, et al. Ceramide synthase 5 mediates lipid-induced autophagy and hypertrophy in cardiomyocytes. J Clin Investig. (2012) 122:3919-30. doi: 10.1172/JCI63888

11. Rodriguez-Cuenca S, Barbarroja N, Vidal-Puig A. Dihydroceramide desaturase 1, the gatekeeper of ceramide induced lipotoxicity. Biochim Biophys Acta. (2015) 1851:40-50. doi: 10.1016/j.bbalip.2014.09.021 
12. Siddique MM, Li Y, Chaurasia B, Kaddai VA, Summers SA. Dihydroceramides: from bit players to lead actors. J Biol Chem. (2015) 290:15371-9. doi: 10.1074/jbc.R115.653204

13. Chaurasia B, Tippetts TS, Monibas RM, Liu J, Li Y, Wang L, et al. Targeting a ceramide double bond improves insulin resistance and hepatic steatosis. Science. (2019) 365:386-92. doi: 10.1126/science.aav3722

14. Kitatani K, Sheldon K, Rajagopalan V, Anelli V, Jenkins RW, Sun Y, et al. Involvement of acid beta-glucosidase 1 in the salvage pathway of ceramide formation. J Biol Chem. (2009) 284:12972-8. doi: 10.1074/jbc.M802790200

15. Claus RA, Dorer MJ, Bunck AC, Deigner HP. Inhibition of sphingomyelin hydrolysis: targeting the lipid mediator ceramide as a key regulator of cellular fate. Curr Med Chem. (2009) 16:1978-2000. doi: 10.2174/092986709788682182

16. Holland WL, Miller RA, Wang ZV, Sun K, Barth BM, Bui HH, et al. Receptormediated activation of ceramidase activity initiates the pleiotropic actions of adiponectin. Nat Med. (2011) 17:55-63. doi: 10.1038/nm.2277

17. Holland WL, Xia JY, Johnson JA, Sun K, Pearson MJ, Sharma AX, et al. Inducible overexpression of adiponectin receptors highlight the roles of adiponectin-induced ceramidase signaling in lipid and glucose homeostasis. Mol Metab. (2017) 6:267-75. doi: 10.1016/j.molmet.2017.01.002

18. Buechler C, Wanninger J, Neumeier M. Adiponectin, a key adipokine in obesity related liver diseases. World J Gastroenterol. (2011) 17:2801-11. doi: 10.3748/wjg.v17.i23.2801

19. Lemoine M, Ratziu V, Kim M, Maachi M, Wendum D, Paye F, et al. Serum adipokine levels predictive of liver injury in non-alcoholic fatty liver disease. Liver Int. (2009) 29:1431-8. doi: 10.1111/j.1478-3231.2009.02022.x

20. Matsuzawa Y. Adiponectin: a key player in obesity related disorders. Curr Pharm Des. (2010) 16:1896-901. doi: 10.2174/138161210791208893

21. Fukushima J, Kamada Y, Matsumoto H, Yoshida Y, Ezaki H, Takemura T, et al. Adiponectin prevents progression of steatohepatitis in mice by regulating oxidative stress and Kupffer cell phenotype polarization. Hepatol Res. (2009) 39:724-38. doi: 10.1111/j.1872-034X.2009.00509.x

22. Matsumoto H, Tamura S, Kamada Y, Kiso S, Fukushima J, Wada A, et al. Adiponectin deficiency exacerbates lipopolysaccharide/D-galactosamineinduced liver injury in mice. World J Gastroenterol. (2006) 12:33528. doi: 10.3748/wjg.v12.i21.3352

23. Angulo $\mathrm{P}$, Kleiner DE, Dam-Larsen S, Adams LA, Bjornsson ES, Charatcharoenwitthaya $P$, et al. Liver fibrosis, but no other histologic features, is associated with long-term outcomes of patients with nonalcoholic fatty liver disease. Gastroenterology. (2015) 149:389-97.e10. doi: 10.1053/j.gastro.2015.04.043

24. Postic C, Girard J. Contribution of de novo fatty acid synthesis to hepatic steatosis and insulin resistance: lessons from genetically engineered mice. $J$ Clin Investig. (2008) 118:829-38. doi: 10.1172/JCI34275

25. Choi SS, Diehl AM. Hepatic triglyceride synthesis and nonalcoholic fatty liver disease. Curr Opin Lipidol. (2008) 19:295-300. doi: 10.1097/MOL.0b013e3282ff5e55

26. Yamaguchi K, Yang L, McCall S, Huang J, Yu XX, Pandey SK, et al. Inhibiting triglyceride synthesis improves hepatic steatosis but exacerbates liver damage and fibrosis in obese mice with nonalcoholic steatohepatitis. Hepatology. (2007) 45:1366-74. doi: 10.1002/hep.21655

27. Li ZZ, Berk M, McIntyre TM, Feldstein AE. Hepatic lipid partitioning and liver damage in nonalcoholic fatty liver disease: role of stearoyl-CoA desaturase. J Biol Chem. (2009) 284:5637-44. doi: 10.1074/jbc.M807616200

28. Yang RX, Pan Q, Liu XL, Zhou D, Xin FZ, Zhao ZH, et al. Therapeutic effect and autophagy regulation of myriocin in nonalcoholic steatohepatitis. Lipids Health Dis. (2019) 18:179. doi: 10.1186/s12944-019-1118-0

29. Alkhouri N, Dixon LJ, Feldstein AE. Lipotoxicity in nonalcoholic fatty liver disease: not all lipids are created equal. Exp Rev Gastroenterol Hepatol. (2009) 3:445-51. doi: 10.1586/egh.09.32

30. Luukkonen PK, Zhou Y, Sadevirta S, Leivonen M, Arola J, Oresic M, et al. Hepatic ceramides dissociate steatosis and insulin resistance in patients with non-alcoholic fatty liver disease. J Hepatol. (2016) 64:116775. doi: 10.1016/j.jhep.2016.01.002

31. Neuschwander-Tetri BA. Hepatic lipotoxicity and the pathogenesis of nonalcoholic steatohepatitis: the central role of nontriglyceride fatty acid metabolites. Hepatology. (2010) 52:774-88. doi: 10.1002/hep.23719
32. Promrat K, Longato L, Wands JR, de la Monte SM. Weight loss amelioration of non-alcoholic steatohepatitis linked to shifts in hepatic ceramide expression and serum ceramide levels. Hepatol Res. (2011) 41:75462. doi: 10.1111/j.1872-034X.2011.00815.x

33. Wasilewska N, Bobrus-Chociej A, Harasim-Symbor E, Tarasow E, Wojtkowska M, Chabowski A, et al. Increased serum concentration of ceramides in obese children with nonalcoholic fatty liver disease. Lipids Health Dis. (2018) 17:216. doi: 10.1186/s12944-018-0855-9

34. Montandon SA, Somm E, Loizides-Mangold U, de Vito C, Dibner C, Jornayvaz FR. Multi-technique comparison of atherogenic and MCD NASH models highlights changes in sphingolipid metabolism. Sci Rep. (2019) 9:16810. doi: 10.1038/s41598-019-53346-4

35. Simon J, Ouro A, Ala-Ibanibo L, Presa N, Delgado TC, MartinezChantar ML. Sphingolipids in non-alcoholic fatty liver disease and hepatocellular carcinoma: ceramide turnover. Int J Mol Sci. (2019) 21:40. doi: 10.3390/ijms21010040

36. Montefusco DJ, Allegood JC, Spiegel S, Cowart LA. Non-alcoholic fatty liver disease: insights from sphingolipidomics. Biochem Biophys Res Commun. (2018) 504:608-16. doi: 10.1016/j.bbrc.2018.05.078

37. Luukkonen PK, Sadevirta S, Zhou Y, Kayser B, Ali A, Ahonen L, et al. Saturated fat is more metabolically harmful for the human liver than unsaturated fat or simple sugars. Diabetes Care. (2018) 41:17329. doi: $10.2337 / \mathrm{dc} 18-0071$

38. Xia JY, Holland WL, Kusminski CM, Sun K, Sharma AX, Pearson MJ, et al. Targeted induction of ceramide degradation leads to improved systemic metabolism and reduced hepatic steatosis. Cell Metab. (2015) 22:26678. doi: 10.1016/j.cmet.2015.06.007

39. Jiang C, Xie C, Li F, Zhang L, Nichols RG, Krausz KW, et al. Intestinal farnesoid X receptor signaling promotes nonalcoholic fatty liver disease. J Clin Investig. (2015) 125:386-402. doi: 10.1172/JCI76738

40. Raichur S, Wang ST, Chan PW, Li Y, Ching J, Chaurasia B, et al. CerS2 haploinsufficiency inhibits beta-oxidation and confers susceptibility to dietinduced steatohepatitis and insulin resistance. Cell Metab. (2014) 20:68795. doi: 10.1016/j.cmet.2014.09.015

41. Powell DJ, Hajduch E, Kular G, Hundal HS. Ceramide disables 3phosphoinositide binding to the pleckstrin homology domain of protein kinase B (PKB)/Akt by a PKCzeta-dependent mechanism. Mol Cell Biol. (2003) 23:7794-808. doi: 10.1128/MCB.23.21.7794-7808.2003

42. Moslehi A, Hamidi-Zad Z. Role of SREBPs in liver diseases: a mini-review. J Clin Transl Hepatol. (2018) 6:332-8. doi: 10.14218/JCTH.2017.00061

43. Worgall TS, Juliano RA, Seo T, Deckelbaum RJ. Ceramide synthesis correlates with the posttranscriptional regulation of the sterol-regulatory element-binding protein. Arterioscler Thromb Vasc Biol. (2004) 24:9438. doi: 10.1161/01.atv.0000125703.20434.4d

44. Chen TC, Lee RA, Tsai SL, Kanamaluru D, Gray NE, Yiv N, et al. An ANGPTL4-ceramide-protein kinase Czeta axis mediates chronic glucocorticoid exposure-induced hepatic steatosis and hypertriglyceridemia in mice. J Biol Chem. (2019) 294:9213-24. doi: 10.1074/jbc.RA118.006259

45. Neuschwander-Tetri BA. Non-alcoholic fatty liver disease. BMC Med. (2017) 15:45. doi: 10.1186/s12916-017-0806-8

46. Dulai PS, Singh S, Patel J, Soni M, Prokop LJ, Younossi Z, et al. Increased risk of mortality by fibrosis stage in nonalcoholic fatty liver disease: systematic review and meta-analysis. Hepatology. (2017) 65:155765. doi: 10.1002/hep. 29085

47. Manco M. Insulin resistance and NAFLD: a dangerous liaison beyond the genetics. Children. (2017) 4:74. doi: 10.3390/children4080074

48. Puri P, Wiest MM, Cheung O, Mirshahi F, Sargeant C, Min HK, et al. The plasma lipidomic signature of nonalcoholic steatohepatitis. Hepatology. (2009) 50:1827-38. doi: 10.1002/hep.23229

49. Puri P, Baillie RA, Wiest MM, Mirshahi F, Choudhury J, Cheung O, et al. A lipidomic analysis of nonalcoholic fatty liver disease. Hepatology. (2007) 46:1081-90. doi: 10.1002/hep.21763

50. Jiang M, Li C, Liu Q, Wang A, Lei M. Inhibiting ceramide synthesis attenuates hepatic steatosis and fibrosis in rats with non-alcoholic fatty liver disease. Front Endocrinol. (2019) 10:665. doi: 10.3389/fendo.2019.00665

51. Kurek K, Piotrowska DM, Wiesiolek-Kurek P, Lukaszuk B, Chabowski A, Gorski J, et al. Inhibition of ceramide de novo synthesis reduces liver lipid 
accumulation in rats with nonalcoholic fatty liver disease. Liver Int. (2014) 34:1074-83. doi: 10.1111/liv.12331

52. Ichi I, Nakahara K, Fujii K, Iida C, Miyashita Y, Kojo S. Increase of ceramide in the liver and plasma after carbon tetrachloride intoxication in the rat. J Nutr Sci Vitaminol. (2007) 53:53-6. doi: 10.3177/jnsv.53.53

53. Akazawa $\mathrm{Y}$, Nakao K. To die or not to die: death signaling in nonalcoholic fatty liver disease. J Gastroenterol. (2018) 53:893-906. doi: 10.1007/s00535-018-1451-5

54. Obeid LM, Linardic CM, Karolak LA, Hannun YA. Programmed cell death induced by ceramide. Science. (1993) 259:176971. doi: $10.1126 /$ science. 8456305

55. Hernandez-Corbacho MJ, Salama MF, Canals D, Senkal CE, Obeid LM. Sphingolipids in mitochondria. Biochim Biophys Acta. (2017) 1862:5668. doi: 10.1016/j.bbalip.2016.09.019

56. Arora AS, Jones BJ, Patel TC, Bronk SF, Gores GJ. Ceramide induces hepatocyte cell death through disruption of mitochondrial function in the rat. Hepatology. (1997) 25:958-63. doi: 10.1002/hep.510250428

57. Ganesan V, Perera MN, Colombini D, Datskovskiy D, Chadha K, Colombini M. Ceramide and activated Bax act synergistically to permeabilize the mitochondrial outer membrane. Apoptosis. (2010) 15:553-62. doi: 10.1007/s10495-009-0449-0

58. Dadsena S, Bockelmann S, Mina JGM, Hassan DG, Korneev S, Razzera G, et al. Ceramides bind VDAC2 to trigger mitochondrial apoptosis. Nat Commun. (2019) 10:1832. doi: 10.1038/s41467-019-09654-4

59. Siskind LJ, Kolesnick RN, Colombini M. Ceramide forms channels in mitochondrial outer membranes at physiologically relevant concentrations. Mitochondrion. (2006) 6:118-25. doi: 10.1016/j.mito.2006.03.002

60. Siskind LJ, Davoody A, Lewin N, Marshall S, Colombini M. Enlargement and contracture of C2-ceramide channels. Biophys J. (2003) 85:156075. doi: 10.1016/S0006-3495(03)74588-3

61. Siskind LJ, Kolesnick RN, Colombini M. Ceramide channels increase the permeability of the mitochondrial outer membrane to small proteins. J Biol Chem. (2002) 277:26796-803. doi: 10.1074/jbc.M200754200

62. Siskind LJ, Colombini M. The lipids C2- and C16-ceramide form large stable channels. Implications for apoptosis. J Biol Chem. (2000) 275:386404. doi: 10.1074/jbc.C000587200

63. Zhou H, Summers SA, Birnbaum MJ, Pittman RN. Inhibition of Akt kinase by cell-permeable ceramide and its implications for ceramide-induced apoptosis. J Biol Chem. (1998) 273:16568-75. doi: 10.1074/jbc.273.26.16568

64. Summers SA, Garza LA, Zhou H, Birnbaum MJ. Regulation of insulinstimulated glucose transporter GLUT4 translocation and Akt kinase activity by ceramide. Mol Cell Biol. (1998) 18:5457-64. doi: 10.1128/MCB.18.9.5457

65. Shmarakov IO, Jiang H, Liu J, Fernandez EJ, Blaner WS. Hepatic stellate cell activation: a source for bioactive lipids. Biochim Biophys Acta. (2019) 1864:629-42. doi: 10.1016/j.bbalip.2019.02.004

66. Nikolova-Karakashian M. Sphingolipids at the crossroads of NAFLD and senescence. Adv Cancer Res. (2018) 140:15590. doi: 10.1016/bs.acr.2018.05.002

67. Shea BS, Tager AM. Sphingolipid regulation of tissue fibrosis. Open Rheumatol J. (2012) 6:123-9. doi: 10.2174/1874312901206010123

68. Sato M, Markiewicz M, Yamanaka M, Bielawska A, Mao C, Obeid LM, et al. Modulation of transforming growth factor-beta (TGF-beta) signaling by endogenous sphingolipid mediators. J Biol Chem. (2003) 278:927682. doi: 10.1074/jbc.M211529200

69. Chen Q, Lee CE, Denard B, Ye J. Sustained induction of collagen synthesis by TGF-beta requires regulated intramembrane proteolysis of CREB3L1. PLoS ONE. (2014) 9:e108528. doi: 10.1371/journal.pone.0108528

70. Chen Q, Denard B, Lee CE, Han S, Ye JS, Ye J. Inverting the topology of a transmembrane protein by regulating the translocation of the first transmembrane helix. Mol Cell. (2016) 63:567-78. doi: 10.1016/j.molcel.2016.06.032

71. Denard B, Lee C, Ye J. Doxorubicin blocks proliferation of cancer cells through proteolytic activation of CREB3L1. Elife. (2012) 1:e00090. doi: 10.7554/eLife.00090

72. Wu S, Wu F, Ding Y, Hou J, Bi J, Zhang Z. Association of non-alcoholic fatty liver disease with major adverse cardiovascular events: a systematic review and meta-analysis. Sci Rep. (2016) 6:33386. doi: 10.1038/srep33386
73. Tana C, Ballestri S, Ricci F, Di Vincenzo A, Ticinesi A, Gallina S, et al. Cardiovascular risk in non-alcoholic fatty liver disease: mechanisms and therapeutic implications. Int J Environ Res Public Health. (2019) 16:3104. doi: 10.3390/ijerph16173104

74. Anroedh S, Hilvo M, Akkerhuis KM, Kauhanen D, Koistinen K, Oemrawsingh $\mathrm{R}$, et al. Plasma concentrations of molecular lipid species predict long-term clinical outcome in coronary artery disease patients. J Lipid Res. (2018) 59:1729-37. doi: 10.1194/jlr.P081281

75. de Carvalho LP, Tan SH, Ow GS, Tang Z, Ching J, Kovalik JP, et al. Plasma ceramides as prognostic biomarkers and their arterial and myocardial tissue correlates in acute myocardial infarction. JACC Basic Transl Sci. (2018) 3:163-75. doi: 10.1016/j.jacbts.2017.12.005

76. Havulinna AS, Sysi-Aho M, Hilvo M, Kauhanen D, Hurme R, Ekroos K, et al. Circulating ceramides predict cardiovascular outcomes in the populationbased FINRISK 2002 cohort. Arterioscler Thromb Vasc Biol. (2016) 36:242430. doi: 10.1161/ATVBAHA.116.307497

77. Hilvo M, Meikle PJ, Pedersen ER, Tell GS, Dhar I, Brenner H, et al. Development and validation of a ceramide- and phospholipid-based cardiovascular risk estimation score for coronary artery disease patients. Eur Heart J. (2019) 41:371-80. doi: 10.1093/eurheartj/ehz387

78. Poss AM, Maschek JA, Cox JE, Hauner BJ, Hopkins PN, Hunt SC, et al. Machine learning reveals serum sphingolipids as cholesterol-independent biomarkers of coronary artery disease. J Clin Invest. (2019) 130:136376. doi: 10.1172/JCI131838

79. Tarasov K, Ekroos K, Suoniemi M, Kauhanen D, Sylvanne T, Hurme R, et al. Molecular lipids identify cardiovascular risk and are efficiently lowered by simvastatin and PCSK9 deficiency. J Clin Endocrinol Metab. (2014) 99:E4552. doi: 10.1210/jc.2013-2559

80. Lemaitre RN, Yu C, Hoofnagle A, Hari N, Jensen PN, Fretts AM, et al. Circulating sphingolipids, insulin, HOMA-IR, and HOMA-B: the strong heart family study. Diabetes. (2018) 67:1663-72. doi: 10.2337/db17-1449

81. Thorens B, Rodriguez A, Cruciani-Guglielmacci C, Wigger L, Ibberson M, Magnan C. Use of preclinical models to identify markers of type 2 diabetes susceptibility and novel regulators of insulin secretion - A step towards precision medicine. Mol Metab. (2019) 27S:S147-54. doi: 10.1016/j.molmet.2019.06.008

82. Wigger L, Cruciani-Guglielmacci C, Nicolas A, Denom J, Fernandez N, Fumeron F, et al. Plasma dihydroceramides are diabetes susceptibility biomarker candidates in mice and humans. Cell Rep. (2017) 18:226979. doi: 10.1016/j.celrep.2017.02.019

83. Liu DJ, Peloso GM, Yu H, Butterworth AS, Wang X, Mahajan A, et al. Exomewide association study of plasma lipids in $>300,000$ individuals. Nat Genet. (2017) 49:1758-66. doi: 10.1038/ng.3977

84. Simons N, Isaacs A, Koek GH, Kuc S, Schaper NC. Brouwers PNPLA3 M, TM6SF2, and MBOAT7 genotypes and coronary artery disease. Gastroenterology. (2017) 152:912-3. doi: 10.1053/j.gastro.2016.12.020

85. Chen L-Z, Xin Y-N, Geng N, Jiang M, Zhang D-D, Xuan S-Y. PNPLA3 I148M variant in nonalcoholic fatty liver disease: demographic and ethnic characteristics and the role of the variant in nonalcoholic fatty liver fibrosis. World J Gastroenterol. (2015) 21:794-802. doi: 10.3748/wjg.v21. i3.794

86. Summers SA. Ceramides: nutrient signals that drive hepatosteatosis. J Lipid Atheroscler. (2020) 9:50-65. doi: 10.12997/jla.2020.9.1.50

Conflict of Interest: SS is a consultant and shareholder with Centaurus Therapeutics.

The remaining author declares that the research was conducted in the absence of any commercial or financial relationships that could be construed as a potential conflict of interest.

Copyright (c) 2020 Poss and Summers. This is an open-access article distributed under the terms of the Creative Commons Attribution License (CC BY). The use, distribution or reproduction in other forums is permitted, provided the original author(s) and the copyright owner(s) are credited and that the original publication in this journal is cited, in accordance with accepted academic practice. No use, distribution or reproduction is permitted which does not comply with these terms. 